\title{
DOMAINS OF VARIABILITY FOR UNIVALENT POLYNOMIALS
}

\author{
V. F. COWLING AND W. C. ROYSTER ${ }^{1}$
}

1. Introduction. In the present note we are concerned with determining necessary and sufficient conditions bearing upon the numbers $a_{2}$ and $a_{3}$ in order that the polynomial $f(z)=z+a_{2} z^{2}+a_{3} z^{3}$ be univalent in the unit disk $|z|<1$. In particular we find the precise domain of variability of $a_{2}$ and $a_{3}$ for the case in which $a_{2}$ and $a_{3}$ are real. In the complex case we characterize the domain of variability by determining a family of parallel cross sections.

2. The principal result. We solve the problem for real coefficients first since the solution in the real case is helpful in determining the solution in the complex case. Our procedure is based on the following theorem whose proof is immediate.

THEOREM 1. A necessary and sufficient condition that $f(z)=\sum_{n=1}^{\infty} a_{n} z^{n}$, $a_{1}=1$, be univalent in $|z|<1$, is that for all $\alpha$ on $0 \leqq|\alpha| \leqq 1, \alpha \neq 1$

$$
\phi_{\alpha}(z)=\frac{z}{f(z)-f(\alpha z)}=\sum_{0}^{\infty} b_{n}(\alpha) z^{n}
$$

be regular in the unit circle.

N.B. We may restrict our attention to $|\alpha|=1, \alpha \neq 1$ if we wish. Now

$$
\phi_{\alpha}(z)=1 / \sum_{1}^{\infty} a_{n}\left(1-\alpha^{n}\right) z^{n-1}=\sum_{0}^{\infty} b_{n}(\alpha) z^{n}
$$

or

$$
1=\sum_{1}^{\infty} a_{n}\left(1-\alpha^{n}\right) z^{n-1} \sum_{0}^{\infty} b_{n} z^{n}=\sum_{0}^{\infty} c_{n} z^{n}
$$

where for $n=1,2, \cdots$ we have

$$
c_{n}=b_{n} a_{1}(1-\alpha)+b_{n-1} a_{2}\left(1-\alpha^{2}\right)+\cdots+b_{0} a_{n+1}\left(1-\alpha^{n+1}\right) .
$$

In detail this yields

Received by the editors October 3, 1966 and, in revised form, March 21, 1967.

1 The research of the first named author was supported in part by National Science Foundation Grant GP-4219 and of the second named author by National Science Foundation Grant GP-3824. 


$$
\begin{aligned}
1= & a_{1}(1-\alpha) b_{0} \\
0= & a_{2}\left(1-\alpha^{2}\right) b_{0}+a_{1}(1-\alpha) b_{1} \\
0= & a_{3}\left(1-\alpha^{3}\right) b_{0}+a_{2}\left(1-\alpha^{2}\right) b_{1}+a_{1}(1-\alpha) b_{2} \\
& \vdots \\
0 & : a_{n}\left(1-\alpha^{n}\right) b_{0}+a_{n-1}\left(1-\alpha^{n-1}\right) b_{1}+\cdots+a_{1}(1-\alpha) b_{n-1} .
\end{aligned}
$$

The $n$ equations in the $n$ unknowns $b_{0}, b_{1}, \cdots, b_{n-1}$ have as solution for $b_{n-1}$

$$
b_{n-1}=c_{n}^{1} / \Delta
$$

where

$$
\Delta=\left|\begin{array}{rrr}
a_{1}(1-\alpha) & \\
& a_{1}(1-\alpha) & \\
0 & & a_{1}(1-\alpha)
\end{array}\right|=a_{1}^{n}(1-\alpha)^{n}
$$

and

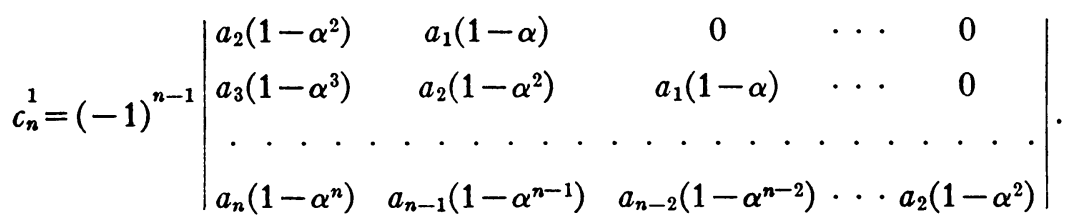

So if we set $a_{1}=1$, divide the columns by $1-\alpha$ and set

$$
p_{n}=p_{n}(\alpha)=1+\alpha+\cdots+\alpha^{n-1}, \quad p_{1}(\alpha)=1
$$

for $n=1,2, \cdots$ we have

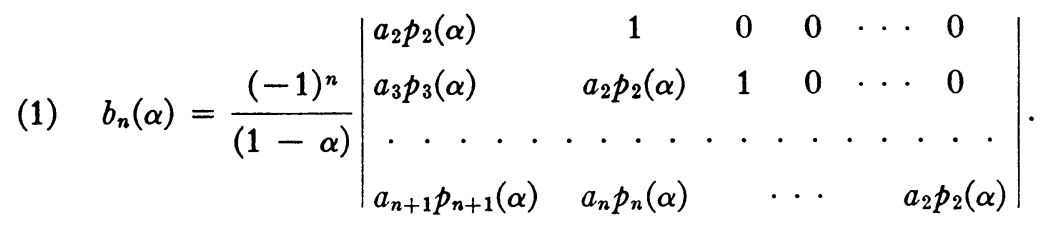

Now a necessary and sufficient condition that $\phi_{\alpha}(z)$ be regular in the unit circle for all $\alpha$ satisfying $|\alpha|=1, \alpha \neq 1$ is that

$$
\operatorname{Lim} \operatorname{Sup}\left(\left|b_{n}(\alpha)\right|\right)^{1 / n} \leqq 1 \text { for all } \alpha,|\alpha|=1, \alpha \neq 1 .
$$

Consider for the moment the trivial example of $f(z)=z+a_{2} z^{2}$, i.e., 
the case $a_{n}=0, n \geqq 3$. Here $b_{n}(\alpha)=(-1)^{n}(1-\alpha)^{-1} a_{2}^{n}(1+\alpha)^{n}$. So for fixed $\alpha$

$\left(\left|b_{n}(\alpha)\right|\right)^{1 / n}=|1-\alpha|^{-1 / n}\left|a_{2}\right||1+\alpha| \rightarrow\left|a_{2}\right||1-\alpha|$ as $n \rightarrow \infty$.

Then as $\alpha \rightarrow 1$ we get $\left|a_{2}\right| \leqq 1 / 2$ as a necessary and sufficient condition for univalence in $|z|<1$. It might be thought that an application of the Hadamard determinant inequality to the case of the general cubic would prove effective. However the following calculation shows this not to be the case. Using the Hadamard theorem one gets

$$
\left|b_{n}(\alpha)\right| \leqq|1-\alpha|^{-1}\left[1+\left|a_{2}^{2} p_{2}^{2}\right|+\left|a_{3}^{2} p_{3}^{2}\right|\right]^{n / 2} .
$$

From this however one gets only that $1+4\left|a_{2}\right|^{2}+9\left|a_{3}\right|^{2} \leqq 1$ as a sufficient condition for univalence, i.e. $f(z)=z$ is univalent in $|z|<1$.

We now return to equation (1) and note that if we denote the determinant in the right-hand member by $R_{n}$ then in the special case $a_{n}=0, n>3$, we have

$$
R_{n}-a_{2} p_{2}(\alpha) R_{n-1}+a_{3} p_{3}(\alpha) R_{n-2}=0 .
$$

From this one may easily show that

$$
\begin{aligned}
R_{n}= & C_{1}\left[\left(a_{2} p_{2}+\left(a_{2}^{2} p_{2}^{2}-4 a_{3} p_{3}\right)^{1 / 2}\right) / 2\right]^{n} \\
& +C_{2}\left[\left(a_{2} p_{2}-\left(a_{2}^{2} p_{2}^{2}-4 a_{3} p_{3}\right)^{1 / 2}\right) / 2\right]^{n}
\end{aligned}
$$

where the values of the constants $C_{1}$ and $C_{2}$ are unimportant. An application of inequality (2) combined with the implications of equations (1) and (3) reveal the following theorem.

THEOREM 2. A necessary and sufficient condition bearing on the complex numbers $a_{2}$ and $a_{3}$ in order that $z+a_{2} z^{2}+a_{3} z^{3}$ be univalent in $|z|<1$ is that

$$
\left|a_{2} p_{2}(\alpha) \pm\left(a_{2}^{2} p_{2}^{2}(\alpha)-4 a_{3} p_{3}(\alpha)\right)^{1 / 2}\right| \leqq 2
$$

for all $\alpha$ satisfying $|\alpha|=1, \alpha \neq 1$.

Note first that if $a_{2}=0$ the inequalities (4) imply $\left|a_{3}\right| \leqq 1 / 3$ while if $a_{3}=0$ the inequalities (4) imply $\left|a_{2}\right| \leqq 1 / 2$. If $p_{2}=0$, i.e., $\alpha=-1$ we have $\left|a_{3}\right| \leqq 1$. Suppose now that $a_{2} p_{2} \neq 0$. Then (4) becomes

$$
\left|a_{2} p_{2}\right|\left|1 \pm 1-\left(4 a_{3} p_{3} / a_{2}^{2} p_{2}^{2}\right)^{1 / 2}\right| \leqq 2 .
$$

A simple calculation reveals that upon setting $\alpha=\exp (i \theta)$,

$$
p_{3} / p_{2}^{2}=1-1 / 4 \cos ^{2}(\theta / 2) \text {. }
$$


Set $\cos (\theta / 2)=\gamma$ and

$$
1-1 / 4 \cos ^{2}(\theta / 2)=1-1 / 4 \gamma^{2}=A / 4 .
$$

Then (5) becomes

$$
\left|a_{2} \gamma\right|\left|1 \pm\left(1-A a_{3} / a_{2}^{2}\right)^{1 / 2}\right| \leqq 1 .
$$

We now spare the reader the details of a lengthy but straightforward analysis of the inequalities (6) for the case of real coefficients. One can obtain these results also from an analysis of the domain of variability for complex coefficients which is discussed in the next section. One considers separately the cases $1-A a_{3} / a_{2}^{2} \geqq 0$ and $1-A a_{3} / a_{2}^{2}<0$. One then plots $a_{3}$ vertically and $a_{2}$ horizontally. This analysis leads to families of half-planes whose intersection determines a convex region $V$ with the property $\left(a_{2}, a_{3}\right) \in V$ is a necessary and sufficient condition for the inequalities (6) to hold and hence for $z+a_{2} z^{2}+a_{3} z^{3}$ to be univalent in $|z|<1$. The equations of the boundary of $V, \partial V$, to be given below are determined in part by finding the envelope of the families of lines bounding the half-planes mentioned above. It is then shown that the domain $V$ of variability of the coefficients is symmetric with respect to the $a_{3}$ axis. The boundary of $V$ in the right half-plane consists of that portion of the line $2 a_{2}-3 a_{3}=1$ between the points $(0,-1 / 3)$ and $(4 / 5,1 / 5)$; the arc of the ellipse $a_{2}^{2}+4\left(a_{3}-1 / 2\right)^{2}=1$ between the points $(4 / 5,1 / 5)$ and $((2 \sqrt{ } 2) / 3,1 / 3)$ and finally that portion of the line $a_{3}=1 / 3$ between the points $((2 \sqrt{ } 2) / 3,1 / 3)$ and $(0,1 / 3)$. In each case mentioned above the endpoints are to be included. We note that the point $((2 \sqrt{ } 2) / 3,1 / 3)$ on the boundary yields the greatest value of $a_{2}$ and $a_{3}$. Thus the extremal polynomial is $z+(2 \sqrt{ } 2) z^{2} / 3+z^{3} / 3$.

If one considers other boundary functions, that is, functions corresponding to $\left(a_{2}, a_{3}\right)$ where $\left(a_{2}, a_{3}\right) \in \partial V$ one finds that along that portion of $\partial V$ given by $a_{3}=1 / 3, f^{\prime}(z)$ has two distinct zeros on $|z|=1$; hence $f$ maps $|z| \leqq 1$ onto a region $D$ whose boundary has two cusps; on the portion of $\partial V$ given by $2 a_{2}-3 a_{3}=1, f^{\prime}(z)$ has only one zero on $|z|=1$; hence the boundary of $D$ has only one cusp; on that portion of $\partial V$ given by $a_{2}^{2}+4\left(a_{3}-1 / 2\right)^{2}=1, f^{\prime}(z)$ has no zeros on $|z|=1$ and the boundary of $D$ is the conformal image of $|z|=1$.

3. Domain of variability for complex coefficients. We wish to describe the boundary of $V_{c}$, the domain of variability for $\left(a_{2}, a_{3}\right)$ where $a_{2}$ and $a_{3}$ are complex. Actually this domain is four-dimensional, but its rotational property makes it possible to define its structure from three-dimensional cross sections. Indeed, if $f(z)=z+a_{2} z^{2}+a_{3} z^{3}$ 
is univalent in $|z|<1$ then $e^{-i \beta} f\left(e^{i \beta} z\right)=z+a_{2} e^{i \beta} z^{2}+a_{3} e^{2 i \beta_{z} z^{3}}$ is also univalent in $|z|<1$, as well as $\bar{f}(\bar{z})=z+\bar{a}_{2} z^{2}+\bar{a}_{3} z^{3}$. Hence if any one of the points $\left(a_{2}, a_{3}\right),\left(a_{2} e^{i \beta}, a_{3} e^{2 i \beta}\right)$ or $\left(\bar{a}_{2}, \bar{a}_{3}\right)$ belongs to $V_{c}$ then so do the others. If we choose $\beta=\pi$ it follows that the cross section $\operatorname{Im}\left(a_{2}\right)$ $=0$ is symmetric about the planes $\operatorname{Re}\left(a_{2}\right)=0$ and $\operatorname{Im}\left(a_{3}\right)=0$ and thus we may assume $\operatorname{Im}\left(a_{2}\right)=0, \operatorname{Re}\left(a_{2}\right) \geqq 0$.

In order that $f(z)$ be univalent in $|z|<1$ the coefficients $a_{2}$ and $a_{3}$ must satisfy, according to (6), the condition

$$
\left|a_{2} \gamma \pm\left(a_{2}^{2} \gamma^{2}-\left(4 \gamma^{2}-1\right) a_{3}\right)^{1 / 2}\right| \leqq 1
$$

for all $\gamma, 0 \leqq \gamma<1$ and $a_{2} \geqq 0$. Let

$$
W=a_{2} \gamma+\left(a_{2}^{2} \gamma^{2}-\left(4 \gamma^{2}-1\right) a_{3}\right)^{1 / 2},
$$

then $\left(W-a_{2} \gamma\right)^{2}=a_{2}^{2} \gamma^{2}-\left(4 \gamma^{2}-1\right) a_{3}$ and $a_{3}=\left(4 \gamma^{2}-1\right)^{-1}$ $\cdot\left(a_{2}^{2} \gamma^{2}-\left(W-a_{2} \gamma\right)^{2}\right)$.

Condition (7) implies that

$$
\left|a_{2} \gamma \pm\left(W-a_{2} \gamma\right)\right| \leqq 1
$$

which is valid if $W$ lies in the intersection $I$ of two circles of radius one with centers at 0 and $2 a_{2} \gamma$. Since in the nontrivial case $I$ is not empty we see that $a_{2} \gamma<1$.

The image of $I$ under the mapping $w=\left(W-a_{2} \gamma\right)^{2}$ for fixed $a_{2}$ and $\gamma$ is the inner loop of the limaçon given by $w=a_{2}^{2} \gamma^{2}-1$ $+2\left(a_{2} \gamma+\cos \phi\right) e^{i \phi}, 0 \leqq \phi<2 \pi$. Its image in the complex $a_{3}$-plane, $a_{2}$ fixed, is the inner loop of the limaçon

$$
\left(4 \gamma^{2}-1\right) a_{3}=1+2\left(a_{2} \gamma+\cos \phi\right) e^{i \phi} .
$$

This limaçon intersects the real axis in the $a_{3}$-plane at the points $\operatorname{Re}\left(a_{3}\right)=-\left(2 a_{2} \gamma+1\right) /\left(4 \gamma^{2}-1\right),\left(2 a_{2} \gamma-1\right) /\left(4 \gamma^{2}-1\right)$ and $1 /\left(4 \gamma^{2}-1\right)$. The points satisfying the condition $2 a_{2} \gamma-\left(4 \gamma^{2}-1\right) \operatorname{Re}\left(a_{3}\right)=1$ and $\left(4 \gamma^{2}-1\right) \operatorname{Re}\left(a_{3}\right)=1$ determine the inner loop of the limaçon in the plane $a_{2}=$ constant, the sharp end intersecting the plane $\operatorname{Im}\left(a_{3}\right)=0$ at $\left(4 \gamma^{2}-1\right) \operatorname{Re}\left(a_{3}\right)=1$.

The domain of variability is a solid whose cross sections parallel to the plane $a_{2}=0$ are intersections $B$ of inner loops of limaçons given by equation (9). The intersection $B$ is itself an inner loop for $0 \leqq a_{2} \leqq 4 / 5$, for if we consider the cross section of $V_{c}$ in the plane $\operatorname{Im}\left(a_{3}\right)=0$ we see that the lines $2 a_{2}-3 \operatorname{Re}\left(a_{3}\right)=1$ and $3 \operatorname{Re}\left(a_{3}\right)=1$ form the boundary. They correspond to the inner loops with $\gamma=1$. The base of $V_{c}$ in the plane $a_{2}=0$ is a disk $\left|a_{3}\right| \leqq 1 / 3$. For $4 / 5 \leqq a_{2} \leqq 2 \sqrt{ } 2 / 3$ a simple calculus argument yields the equations $a_{2}=0, a_{2}^{2}+4\left(\operatorname{Re}\left(a_{3}\right)-1 / 2\right)^{2}=1, \operatorname{Re}\left(a_{3}\right)$ 
$=1 / 3$ must be the boundary of the cross section of $V_{c}$ in the plane $\operatorname{Im}\left(a_{3}\right)=0$. Hence the cross sections of $V_{c}$ in $a_{2}=b, 4 / 5 \leqq b \leqq(2 \sqrt{ } 2) / 3$, are not inner loops of a limaçon, but are intersections of inner loops.

4. Conclusion. Although it would be of interest to obtain the precise domain of variability for the trinomial $z+a_{2} z^{2}+a_{k} z^{k}$, for $k>3$ the present methods yield only incomplete results in this direction.

Rutgers, The State University,

The Institute for Advanced Study and

The University OF KentuCky 